\title{
EL INGENIERO INDUSTRIAL REQUERIDO EN LA REPUBLICA DOMINICANA
}

\section{EULOGIO SANTAELLA}

El tema a desarrollar resulta ser tan amplio que no puede ser tratado con toda la profundidad que fuera deseable en el corto espacio de una nota como la presente.

En realidad, pensamos que el título de este trabajo pudo ser: "Ingeniería industrial: la gran desconocida". La sugerencia del mismo se debe a que son pocas las personas en nuestro medio que tienen una concepción relativamente acertada sobre qué es en sí la ingeniería industrial y qué se logra mediante su aplicación en la práctica. Tal desconocimiento constituye un hecho paradógico porque hace ya más de una década que comenzaron a retornar a nuestro país grupos de jóvenes dominicanos que se habían formado como ingenieros industriales en universidades extranjeras, sobre todo en Estados Unidos de América, Puerto Rico y México. Asímismo, a partir de 1970, la Universidad Católica Madre y Maestra ha venido graduando un número significativo de ingenieros industriales y el INTEC, por su parte, ha otorgado en tres promociones diferentes alrededor de docena $y$ media de diplomas de post-grado en ingeniería industrial. Merecen ser mencionados aquí, además, los técnicos-medios o tecnólogos en producción; ya que son ellos los profesionales de nivel medio dentro de la ingeniería industrial. ¿Y dónde se hayan todos esos profesionales? La mayor parte, por no decir la casi totalidad de ellos, desarrolla sus actividades en la República Dominicana. Y sin embargo, a pesar de estar aquí, su presencia aparentemente no se hace sentir lo suficiente. ¿A qué se dedican esos ingenieros industriales que por desconocidos re- 
sultan casi imperceptibles en nuestra sociedad? Esta última pregunta ha sido mal formulada expresamente por nosotros, para así tener la oportunidad de aclarar que las actividades a las cuales se dedica un titulado, bien en ingeniería industrial bien en otra profesión cualquiera, no dependen exclusivamente de la orientación y contenido de la carrera que se haya cursado. Por el contrario, en ello influyen poderosamente tanto el medio social y económico como las condiciones personales del profesional. Así pues, en lugar de hablar sobre las actividades a las que están dedicados hoy los ingenieros industriales residentes en el país, lo apropiado consiste en exponer cuáles son, desde un punto de vista general, las actividades que idealmente debería desarrollar un ingeniero industrial, con independencia de la zona geográfica en donde trabaje. Sólo una vez aclarado esto, se contará con una base suficientemente sólida para analizar cuáles características deberá reunir un ingeniero industrial para responder a los requerimientos de la sociedad dominicana tanto de hoy día como del futuro inmediato.

Hecha esta necesaria introducción, se deberá ante todo señalar que las referencias a la ingeniería industrial que aparecen en las publicaciones oficiales dominicanas son bastante escasas. Este hecho nos priva de una valiosa ayuda para este trabajo. Ya que, de existir una "verdad oficial" al respecto, hubiéramos podido utilizar esos datos como marco de referencia para la exposición de nuestras opiniones personales.

Una de las pocas publicaciones oficiales de los últimos años que menciona algo sobre ingeniería industrial es la Plataforma para el desarrollo económico y social de la República Dominicana (1968-1985). ${ }^{1}$ En ella, al hablarse de la etapa de "recuperación nacional y de la reforma agraria". a ser desarrollada entre 1968 y 1970, se indica que: "La formación de técnicos medios, agrícolas e industriales, así como de técnicos pesqueros deberá impulsarse desde el comienzo del período. En un nivel mayor, es indispensable aumentar la formación de ingenieros agrónomos, industriales y veterinarios". ${ }^{2} Y$ más adelante se agrega que, después de los técnicos agrícolas, "en un segundo lugar, en las prioridades, están las necesidades de técnicos industriales de nivel medio y finalmente los técnicos agropecuarios e industriales de nivel superior". 3

Las dos citas anteriores, aunque no permiten sacar una idea concluyente sobre lo que es la ingeniería industrial, sirven al menos para percatarnos de que, al hablarse en la Plataforma de ingenieros industriales, se está haciendo referencia a todos los ingenieros que desempeñan sus labores básicamente en el sector manufacturero, es decir en las industrias. Dentro de una definición tan general entrarían, desde luego, los ingenieros industriales propiamente dichos, que según nuestra concepción son los ingenieros químicos, eléctricos, mecánicos, electrónicos, computacionales, etc. En otras palabras, en la Plataforma se habla de ingenieros industriales como denominación genérica de todos aquellos ingenieros que 
de hecho operan en la manufactura, para diferenciarlos de los ingenieros agrónomos y veterinarios que se desempeñan en los campos agrícola y pecuario respectivamente.

Ahora bien, esta falta de comprensión cabal sobre lo que es la ingeniería industrial no sólo ocurre entre nosotros, sino que se da incluso en países de mayor desarrollo que el nuestro. Esto se debe a que los pioneros de la ingeniería in dustrial se dedicaron a desarrollar y aplicar esa disciplina sin poner mucha atención en definirla.

La confusión que reina sobre el término es tal que la versión castellana de un texto tan conocido como el de William Grant Ireson y Eugene L. Grant no refleja su verdadero título en inglés. En efecto, mientras que el original inglés reza Handbook of industrial engineering and management, la titulación castellana fue Manual de ingeniería económica y organización industrial. ${ }^{4} Y$ para arrojar aún más oscuridad sobre el asunto, el autor del prefacio de la versión castella, Gabriel Zaid, prefiere hablar en él de "ingeniería económica" y no de "ingeniería industrial"; llegando a afirmar que:

Antes de adoptar el adjetivo "economista" para "ingeniero economista", hemos rechazado los de "ingeniero industrial", "ingenie ro comercial", "ingeniero administrador", "ingeniero de métodos", y otros peores. Quien esto escribe tiene la desgracia de ostentar, o mejor dicho, de no poder ostentar su título de ingeniero mecánico administrador (del Instituto Tecnológico de Monterrey, México). Su consuelo es haber tenido un colega consultor, graduado en Chile, que cargaba con el título de ingeniero comercial. En México, y quizás otros países hispanoamericanos, lo de "ingeniero industrial" es tan pardo y dice tan poco que podría usarse para el caso, esperando que llegará a asociársele la significación respectiva. Además, es muy cómodo para tratar con funcionarios o técnicos provenientes de Estados Unidos, porque es la traducción literal de "industrial engineer" $y$ aunque el término no tiene una total aceptación en Estados Unidos, es cada vez más conocido y muy probable que Ilegue a establecerse. Sólo que el término de "ingeniería industrial" tiene en el diccionario una significación muy precisa que discrepa de "industrial engineering", y corresponde en realidad a "plant engineering", que es una cosa muy distinta".

Valga la extensión de la cita que precede para ilustrar la dificultad existente en definir el campo que nos ocupa. Hecha esa salvedad, podemos ya ofrecer nuestra definición de ingeniería industrial que es la aceptada por el Instituto Americano de Ingenieros Industriales y que fuera elaborada en 1955. Tal definición especifica que:

La Ingeniería Industrial tiene que ver con el diseño, mejoramiento, e instalación de sistemas integrados de hombres, materiales y equipo. Hace uso del conocimiento especializado y de las técnicas de las ciencias matemáticas, físicas y sociales, conjuntamente con los principios y métodos del diseño y 

sistemas. ${ }^{6}$

Para aclarar más el tema que nos atañe transcribiremos algunos conceptos que sobre el particular aparecen en el catálogo informativo de la Escuela de Ingeniería y Ciencias Aplicadas de la Universidad de Columbia, Nueva York:

\begin{abstract}
Ingeniería Industrial es la rama de la profesión de ingeniería que diseña, controla, opera y dirige las organizaciones y sistemas productivos. Originalmente, el ingeniero industriál trabajaba en la industria manufacturera y le concernían la eficiencia operativa y el control de los trabajadores. Hoy día, la ingeniería industrial puede ser hallada no sólo en la producción sino en todos los tipos de industria-manufacturera, de distribución, de transporte, comercio, de servicios-y además en todas las clases de organizaciones-administrativas, gubernamentales, o institucionales. Su trabajo también se ha expandido en amplitud, reflejando el desarrollo de las disciplinas de la ingeniería industrial. Sus responsabilidades abarcan desde el diseño de operaciones unitarias hasta la organización de empresas completas; además incluyen especialidades profesionales como control estad ístico de calidad, investigación de operaciones, $y$ otras.
\end{abstract}

Estos conceptos explicitan algo más nuestro tema y nos sirven como guía para afirmar que, por lo menos en los medios académicos dominicanos, se aceptan muchos títulos como sinónimos o cuasi-sinónimos de "ingeniero industrial". Entre tales títulos se encuentran, entre otros, los siguientes: "ingeniero mecánico-administrador", "ingeniero químico-administrador", "ingeniero industrial-administrador", "ingeniero comercial" según la concepción de los países del Cono Sur del continente americano, "ingeniero de producción" a la usanza germánica o británica, e "ingeniero de métodos".

¿Qué tienen en común todos estos ingenieros que acabamos de mencionar? Sencillamente el hecho de operar ligados a sistemas integrados por materiales, equipos y seres humanos y que tratan de obtener la máxima productividad posible en la operación de tales sistemas. Otra característica común a todos ellos es que a un nivel más o menos rudimentario, hacen uso de técnicas tales como el estudio de métodos y simplificación del trabajo, la medición del trabajo, la localización de plantas, la distribución de maquinaria y otras facilidades físicas en las unidades productivas, el manejo de materiales, la planeación y control de la producción, el control de inventarios, el control de calidad, el establecimiento de normas o estándares, la seguridad industrial, etc. A un nivel más sofisticado, los genieros industriales emplean herramientas altamente "matematizadas" como la "investigación de operaciones".

Creemos que con lo hasta aquí expuesto la idea acerca de lo que es un ingeniero industrial queda menos oscura que al principio. Sin embargo, debemos recalcar, como se hace en el citado catálogo de la Universidad de Columbia, que 
los ingenieros industriales ya han desbordado los recintos de las fábricas y hoy día se encuentran desempeñando importantes tareas en organismos pertenecienel a todos los sectores económicos, no solamente al industrial. En consecuencia, el nombre de "ingeniero industrial" ya no es actualmente útil y significativo. Por lo que en algunos países se utilizan ya nombres tales como "ingeniero de producción" o "ingeniero industrial y de sistemas", entre otros.

Con esto podemos pasar ya al segundo punto que nos propusimos desarrollar y que es el tipo de ingeniero industrial requerido en el momento presente por la República Dominicana.

Tratando de ser breves, analizaremos en primer lugar cuál es, a nuestro juicio, la orientación que debe dársele al "pensum" de la carrera de ingeniería industrial en nuestro país. Hace unos diez años éramos partidarios de que los ingenieros industriales recibieran una formación tan sólida como fuera posible en las materias que constituyen los bloques de ciencias de la ingeniería eléctrica y sobre todo de la mecánica. Las razones para pensar de esa forma eran obvias, pero merecen ser citadas. Se pretendía que el estudiante una vez graduado, al tener una formación flexible y abarcadora, encontrara mayores oportunidades de empleo. Asimismo, en ese entonces, la carrera de ingeniería electro-mecánica no había alcanzado ni remotamente el grado de desarrollo académico y práctico que hoy posee, por lo que un ingeniero industrial con ciertas aptitudes personales podía ejercer con éxito en esa área. Abundan, en efecto, honrosos ejemplos de ingenieros industriales que años atrás tomaron triunfalmente esos derroteros. Pero existe otra razón adicional: la ingeniería industrial era en aquella época, como aún lo es hoy día, menos conocida que las ingenierías eléctrica y mecánica; por lo que un ingeniero industrial estaba mejor capacitado para desenvolverse profesionalmente si al solicitar empleo por primera vez podía realmente decir que su carrera era afĩn de la ingeniería mecánica, y que él poseía sólidos conocimientos sobre ésta.

En cuanto a los cursos propios de ingeniería industrial, creíamos entonces y aún seguimos creyendo que nuestros candidatos a ingenieros industriales deben recibir una fuerte concentración de cursos de lo que podríamos llamar "ingeniería industrial clásica", en contraposición a las más modernas y sofisticadas herramientas que en los últimos años se han venido enseñando a los ingenieros industriales graduados en las universidades de los países desarrollados.

Sin embargo, creemos que en la actualidad no hay razón suficien te ni valedera para que la ingeniería eléctrica y mecánica sigan ocupando un lugar preponderante dentro de la carrera de ingeniería industrial. Lo que hoy se necesita, de acuerdo a nuestra opinión, es una carrera de ingeniería industrial cada vez más "pura" y especializada. Si es que se puede hablar de "pureza" en una carrera 
que, como la ingeniería industrial, es híbrida o multidisciplinaria por antonomasia. Los cursos de ingeniería eléctrica y mecánica deben ser desplazados por cursos sobre gerencia, finanzas, economía y por los de la propia ingeniería industrial.

Sabemos que las características y los problemas de la econom ía dominicana en general y del sector industrial en particular son muy diferentes a los de un país desarrollado. Asimismo, que aunque el sector industrial ha mostrado durante los últimos años en su crecimiento un dinamismo realmente sorprendente, quien visite muchas plantas productoras dominicanas encontrará todavía deficiencias en cosas tan obvias como la distribución de la maquinaria, el manejo de materiales y el diseño de los métodos y de los procesos de transformación. En consecuencia, si queremos ganar la batalla de la producción y de la productividad tenemos que poner en manos de nuestros ingenieros industriales armas similares a las que utilizaron los "expertos en eficiencia" que surgieron en los Estados Unidos de América después de Taylor, el padre de la "administración científica".

Aunque opinamos que las enseñanzas de Taylor y sus discípulos están ya superadas, sin embargo creemos que aún en la actualidad tienen algunos aspectos positivos que ofrecer a países como el nuestro que padecen de graves ineficiencias en todos los sectores productivos.

No debemos pasar por al to esta oportunidad en que hemos mencionado a Taylor, para recordar a nuestros actuales y futuros ingenieros industriales que el más grave problema que afecta a la sociedad dominicana actual está constituido por la situación de desempleo y subempleo que se presenta, con variantes más o menos trágicas, a todo lo largo y ancho del país. La causa de mencionar aquí este problema radica en la existencia de un prejuicio que caricaturiza al ingeniero industrial como un individuo al que se le paga para cancelar trabajadores. Dicho prejuicio tiene, sin embargo, en ocasiones asidero real. En una situación de grave desempleo, ésta se empeoraría aún más si los ingenieros industriales se asignaran a sí mismos el papel de deshauciadores de trabajadores en nombre de una real o supuesta eficiencia.

El ingeniero industrial no debe traicionar su ideal de lograr una mayor eficiencia, pero tampoco debe olvidar que su profesión es sólo un medio y que el hombre es un fin en sí mismo. Además debe tener presente que la eficiencia carece de justificación moral, a menos que los beneficios que de ella se deriven estén equitativamente distribuídos. Aún más, si el ingeniero industrial no desea trillar los caminos del humanismo a ultranza, aún le queda el recurso de dejarse guiar por Fayol y a la hora de buscar ineficiencias fijar su atención en los mandos al tos y medios de las empresas y no sólo a nivel del taller de producción. Si a nivel bajo una empleomanía excesiva o ineficiente es perjudicial, a nivel medio o al to es sencillamente in tolerable. 
Convendrá dedicar ahora un breve comentario al análisis de algunas faltas graves de los ingenieros industriales. Creemos que nuestra falla más notoria la constituye el mutismo y la falta de participación en la definición de las grandes políticas cuya implantación conduzca a la solución de los problemas de nuestro pais. Uno de nuestros deberes incumplidos consiste en no haber impulsado significativamente el crecimiento industrial y económico del país elaborando y promoviendo la creación de nuevos proyectos productivos por parte del sector público o privado, de tal suerte que la mano de obra ociosa encuentre cada vez más fuentes de trabajo. En esta tarea de creación de empleos, la tecnología que se seleccione juega un rol de crucial importancia. Muy pocos ingenieros industriales nos hemos preocupado por difundir la necesidad que existe de estudiar a fondo las implicaciones que en la práctica podrían derivarse de utilizar la llamada "tecnología intermedia" o "tecnología apropiada". Hasta ahora los voceros de esa esperanzadora corriente de pensamiento han sido profesionales de otras áreas del saber.

E. F. Schumacher, en su libro Small is beautiful: economics as if people mattered $^{8}$ ha popularizado la noción de una "tecnología in termedia", la cual según su opinión: "Es vastamente superior a la tecnología primitiva de las edades pasadas, pero al mismo tiempo es mucho más simple, más barata y más libre que la super-tecnología del rico..." $\mathrm{El}$ au tor asimismo afirma que:

La tecnología intermedia también se ajustaría mucho más suavemente en el ambiente relativamente no sofisticado en el cual ha de ser utilizada El equipo sería bastante simple y por tan to inteligible, además de poder recibir mantenimien to $y$ reparación en el propio lugar de uso... (sería) mucho menos dependiente de materias primas de gran pureza o de especificaciones exactas y mucho más adaptable a las fluctuaciones del mercado que el equipo al tamente sofisticado. Los hombres son más fácilmente entrenad os; la supervisión, el control y la organización son más simples y hay mucha menos vulnerabilidad a di-
ficultades imprevistas. 10

Para que no se nos tome por ilusos al hablar de la tecnología intermedia, mencionaremos uno de los muchos ejemplos exitosos de aplicación de "tecnología apropiada" que aparece reseñado en una reciente publicación del Centro de Desarrollo de la OCDE, titulada Appropriate technology; problems and promises ${ }^{1}$. El ejemplo se refiere al caso del empresario filipino que construyó una planta de separación de almidón utilizando como único equipo máquinas lavadoras de segunda mano por valor de 10,000 dólares. El rendimiento de su planta era tan considerable y sus costos de producción tan competitivos, que la planta "eficiente" de medio millón de dólares establecida en la misma región tuvo que ser cerrada.

Por razones de tiempo no podemos hablar en esta oc asión sobre la influen- 
cia que el ingeniero industrial podría tener en el sector agrícola y particularmente en los campos cañeros.

Concluiremos, en cambio, presentando algunas consideraciones sobre la ética en el ejercicio de la profesión de ingeniero. No trataremos de la problemática ligada a los contratos de construcción otorgados por el estado siguiendo la modalidad llamada "de grado a grado", ya que de ese problema se ha dicho bastante. Lo que sí desearíamos indicar es que tanto los ingenieros industriales como los de las otros áreas tenemos el deber de contribuir con nuestro comportamiento profesional, como educadores y como ciudadanos a que cambie la imagen negativa que se tiene del profesional de la ingeniería en amplios sectores de nuestro pueblo. A nadie escapa el que para gran parte de la población dominicana no existen ingenieros industriales, civiles, eléctricos, mecánicos, etc., sino ingenieros a secas, sin ningún calificativo. Así pues, si en algunos sectores sociales se considera, con razón o sin ella, que los ingenieros no son más que timadores, esa mala fama recae sobre todos por igual. $Y$ por más que esa generalización sea injusta, ella nos impedirá nuestro fin último como profesionales que es, a fin de cuentas, coadyuvar al bienestar de nuestro pueblo y de la humanidad.

\section{NOTAS}

1. República Dominicana. Secretariado Técnico de la Presidencia. Oficina Nacional de Planificación. Plataforma para el desarrollo económico y social de la República Dominicana, 1968-1985. Santo Domingo, 1968.604 p.

2. Ibidem., p.86. El subrayado es mío.

3. Ibidem., p.400. El subrayado es mío.

4. William Grant Ireson and Eugene L. Grant Handbook of industrial engineering and management. 2.ed. Englewood Cliffs, N. J., Prentice-Hall, 1971.907 p.

Manual de ingeniería económica y organización industrial. México, CECSA, 1969. $1452 \mathrm{p}$.

5. Idem.

6. Norman N. Barish. "Operations research and industrial engineering: the applied science and its engineering". Operations research, 11(3): 389, May.-Jun. 1963.

7. "Industrial and management engineering". In: Columbia University. School of Engineering and Applied Science. Bulletin 1969-70. New York, 1971. p.54.

8. E. F. Schumacher. Small is beautiful: economics as if people mattered. New York, Harper \& Row, 1975. 290 p. 
9. Ibidem., p.145.

10. Ibidem., p.170.

11. Nicolas léquier. Appropiate technology; problems and promises. Paris, OECD, 1976. $348 \mathrm{p}$. 